\title{
Spider XFEM, an extended finite element variant for partially unknown crack-tip displacement
}

\author{
Elie Chahine* — Patrick Laborde** — Yves Renard*** \\ * Institut de Mathématiques \\ UMR CNRS 5215, GMM INSA Toulouse \\ Complexe scientifique de Rangueil, F-31077 Toulouse cedex 4 \\ elie.chahine@insa-toulouse.fr \\ ** Institut de Mathématiques \\ UMR CNRS 5215, UPS Toulouse 3 \\ 118 route de Narbonne, F-31062 Toulouse cedex 4 \\ patrick.laborde@math.ups-tlse.fr \\ *** Institut Camille Jordan \\ CNRS UMR 5208, INSA de Lyon, Université de Lyon \\ 20 rue Albert Einstein, F-69621 Villeurbanne cedex \\ yves.renard@insa-lyon.fr
}

\begin{abstract}
In this paper, we introduce a new variant of the extended finite element method (Xfem) allowing an optimal convergence rate when the asymptotic displacement is partially unknown at the crack tip. This variant consists in the addition of an adapted discretization of the asymptotic displacement. We give a mathematical result of quasi-optimal a priori error estimate which allows to analyze the potentialities of the method. Some computational tests are provided and a comparison is made with the classical Xfem.

RÉSUMÉ. Dans cet article, nous introduisons une nouvelle variante de la méthode des éléments finis étendus (Xfem) permettant l'obtention d'un taux de convergence optimal lorsque le déplacement asymptotique en pointe de fissure est partiellement inconnu. Cette variante consiste en l'addition d'une discrétisation adaptée du déplacement asymptotique. Nous donnons un résultat mathématique d'estimation d'erreur a priori quasi optimal qui permet d'analyser les potentialités de la méthode. Des tests numériques sont présentés et une comparaison est faite avec la méthode Xfem classique.

KEYWORDS: fracture, Xfem, error estimates, numerical convergence rate.

MOTS-CLÉS : fracture, Xfem, estimation d'erreur, taux de convergence numérique.
\end{abstract}

DOI:10.3166/REMN.17.625-636 @ 2008 Lavoisier, Paris

REMN - 17/2008. Giens 2007, pages 625 to 636 


\section{Introduction}

In order to overcome some difficulties coming from classical finite element strategies (refinement of the mesh around the crack tip, remeshing after crack propagation) many approaches have been developed to make the finite element methods more flexible. In 1973, a nonsmooth enrichment method using a cut-off function for a mesh dependent on the domain geometry is introduced in (Strang et al., 1973). Since then, different approaches had been analyzed such the Pufem (the Partition of Unity Finite Element Method) (Melenk et al., 1996), the Arlequin method (Bendhia, 1998), the Gfem (Generalized Finite Element Method) (Strouboulis et al., 2000), the Xfem (eXtended Finite Element Method) and the patches enrichment approach (Glowinski et al., 2003). Inspired by the Pufem, the Xfem was introduced in (Moës et al., 1999b; Moës et al., 1999a)). It consists on the enrichment of the classical finite element basis by a step function along the crack line to take into consideration the discontinuity of the displacement field and on some nonsmooth functions to represent the asymptotic displacement in a vicinity of the crack tip. This enrichment strategy allows the use of a mesh independent of the crack geometry. Since the introduction of the Xfem, a rapidly growing literature have been produced in order to explore the capabilities of the method and improve its accuracy, two examples of which are (Laborde et al., 2005) and (Béchet et al., 2005).

In this paper, we propose and analyze a new variant of Xfem. It consists in the addition of an adapted patch to the classical Xfem method in order to approximate the asymptotic displacement at the crack tip. There is some similarities with the patches enrichment approach proposed in (Glowinski et al., 2003) but with significant differences. The interest of the method is to avoid the enrichment of the finite element space with the complete asymptotic displacement when the latter is too much complicated or when its complete expression is not available. Only a partial knowledge of the form of the asymptotic displacement is necessary. We give a mathematical result of a priori error estimate which allows to analyze the potentialities of the method. These results are validated by some numerical computations and comparisons with the classical Xfem.

\section{The Spider eXtended Finite Element Method (SpiderXfem)}

We denote $\Omega \subset \mathbb{R}^{2}$ the reference configuration of a cracked linearly isotropic elastic body in plane stress approximation. The boundary of $\Omega$, denoted $\partial \Omega$, is partitioned into three parts $\Gamma_{D}, \Gamma_{N}$ and $\Gamma_{C}$. A Dirichlet condition is prescribed on $\Gamma_{D}$ and a Neumann one on $\Gamma_{N}$ and $\Gamma_{C}$. The part $\Gamma_{C}$ of the boundary is representing the crack (see Fiure 1).

Let $\mathbf{V}=\left\{\mathbf{v} \in H^{1}\left(\Omega, \mathbb{R}^{2}\right) ; \mathbf{v}=0\right.$ on $\left.\Gamma_{D}\right\}$ be the space of admissible displacements and let us define

$$
a(\mathbf{u}, \mathbf{v})=\int_{\Omega} \boldsymbol{\sigma}(\mathbf{u}): \varepsilon(\mathbf{u}) d x, l(\mathbf{v})=\int_{\Omega} \boldsymbol{\xi} \cdot \mathbf{v} d x+\int_{\Gamma_{N}} \boldsymbol{\zeta} \cdot \mathbf{v} d \Gamma,
$$




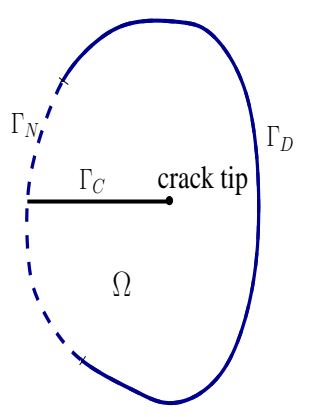

Figure 1. The cracked domain $\Omega$ which represents the reference configuration of a cracked elastic body

$$
\boldsymbol{\sigma}(\mathbf{u})=\lambda \operatorname{tr} \varepsilon(\mathbf{u}) I+2 \mu \varepsilon(\mathbf{u}),
$$

where $\boldsymbol{\sigma}(\mathbf{u})$ is the stress tensor, $\varepsilon(\mathbf{u})$ is the linearized strain tensor, $\boldsymbol{\xi}$ and $\boldsymbol{\zeta}$ are some given force densities on $\Gamma_{N}$ and $\Omega$ respectively, and $\lambda>0, \mu>0$ are the Lamé coefficients (which may have different values on one side and on the other side of the crack for the bi-material case). The elastostatic problem reads as

$$
\text { Find } \mathbf{u} \in \mathbf{V} \text { such that } a(\mathbf{u}, \mathbf{v})=l(\mathbf{v}) \quad \forall \mathbf{v} \in \mathbf{V} .
$$

We suppose that the solution $\mathbf{u}$ to this problem is a sum of a regular part and a nonsmooth part

$$
\mathbf{u}=\mathbf{u}_{r}+\mathbf{u}_{s}
$$

such that $\mathbf{u}_{r}$ is regular in the sense $\mathbf{u}_{r} \in H^{2+\varepsilon}\left(\Omega ; \mathbb{R}^{2}\right)$ for a fixed $\epsilon>0$ (see (Adams, $1975)$ for the definition of $\left.H^{s}\left(\Omega ; \mathbb{R}^{2}\right), s \in \mathbb{R}\right)$ and $\mathbf{u}_{s}$ is of the form

$$
\mathbf{u}_{s}=\left(r^{\alpha} f_{i}(r) g_{i}(\theta)\right)_{i=1,2},
$$

where $(r, \theta)$ are the polar coordinates relatively to the crack tip, $f_{i}, g_{i}, i=1,2$ are some regular functions and $\alpha \geq 1 / 4$.

This assumption is satisfied in the homogeneous case at least when $\boldsymbol{\xi}, \boldsymbol{\zeta}$ are sufficiently smooth, for a straight crack and when the uncracked domain $\bar{\Omega}:=\Omega \cup \Gamma_{C}$ has a regular boundary (see (Grisvard, 1992; Grisvard, 1986)). In this case, for which $\alpha=1 / 2$, the expression of the asymptotic displacement is available in many references such as (Lemaitre et al., 1994). Note that, when $\bar{\Omega}$ admits some corners, some additional nonsmooth displacements may appear at these corners which may also be taken into account with additional enrichment in an Xfem like approach.

The main idea of SpiderXfem is to approximate the nonsmooth behavior around the crack tip of $\Omega$ by another overlapping mesh. We consider a Lagrange finite element method defined on a triangulation $\mathcal{T}^{h}$ of the uncracked domain $\bar{\Omega}$. In accordance with the Xfem method (Moës et al., 1999b), the appropriate degrees of freedom of $\mathcal{T}^{h}$ 
are enriched using a Heaviside function $H$ equal to 1 on one side of the crack and -1 on the other side. This means that the regular part of the displacement field is approximated by a linear combination of the form

$$
\sum_{i \in I} \mathbf{a}_{i} \phi_{i}+\sum_{i \in I_{H}} \mathbf{b}_{i} H \phi_{i}
$$

where $\mathbf{a}_{i} \in \mathbb{R}^{2}, \mathbf{b}_{i} \in \mathbb{R}^{2}, I$ is the set of the indices of the classical finite element nodes, $I_{H}$ is the set of the indices of the nodes enriched by the Heaviside function and $\phi_{i}$ denotes the shapes functions of the scalar finite element method. We define now another rectangular domain $\left.\Omega_{c}=\right]-\pi, \pi[\times] 0, r_{1}[$ in a cartesian coordinate system in $r$ and $\theta$ (see Figure 2). Then, we consider a bi-linear Lagrange finite element method defined on a structured mesh composed of quadrilaterons $Q^{h_{c}}$ of $\Omega_{s}\left(h_{c}\right.$ is the size of the quadrilaterons). In order to take into account the "nonsmooth part" $r^{\alpha}$, the shape functions of this finite element method are multiplied by the term $r^{\alpha}$. Then we apply a geometric transformation to $\Omega_{c}$ defined by

$$
\left\{\begin{array}{l}
x=r \cos \theta+x_{0} \\
y=r \sin \theta+y_{0}
\end{array}\right.
$$

$\left(x_{0}, y_{0}\right)$ being the coordinates of the crack tip. This allows us to have a "circular" mesh denoted $\Omega_{s}$ as depicted in Figure 2. In order to make a smooth transition between the enriched area and the nonenriched one, we introduce a $\mathcal{C}^{2}$ cut-off function $\chi$ which satisfies for $0<r_{0}<r_{1}$

$$
\left\{\begin{array}{l}
\chi(r)=1 \text { if } r<r_{0}, \\
0<\chi(r)<1 \text { if } r_{0}<r<r_{1}, \\
\chi(r)=0 \text { if } r_{1}<r,
\end{array}\right.
$$

which can be, for instance, a piecewise fifth degree polynomial in $r$. The asymptotic displacement at the crack tip is approximated on $\Omega_{s}$ by a linear combination of the form

$$
\sum_{i \in I_{s}} \mathbf{c}_{i} \chi(r) \psi_{i}(r, \theta)
$$

where $\mathbf{c}_{i} \in \mathbb{R}^{2}, I_{s}$ is the set of the indices of the finite element nodes on $\Omega_{s}$ and $\psi_{i}(r, \theta)$ is obtained by applying the geometric transformation to the finite element shape function of the bi-linear finite element method defined on $Q^{h_{c}}$. The shape functions $\psi_{i}$ can be written as follows:

$$
\psi_{i}(r, \theta)=r^{\alpha} p_{j}(r) q_{k}(\theta),
$$

where $p_{j}(r)$ and $q_{k}(\theta)$ are some piecewise first degree polynomial which represent the shape functions of a $P_{1}$ finite element method defined on $\left[0, r_{0}\right]$ and $[0,2 \pi]$, respectively. Finally we overlap $\Omega$ and $\Omega_{s}$ such that the center of $\Omega_{s}$ coincides with the crack tip, and the two sides $\pi$ and $-\pi$ coincides with the crack (Figure 3 ). Thus, the resulting finite element approximation space over $\Omega$ can be written

$$
\mathbf{V}^{h}=\left\{\mathbf{u}^{h}=\sum_{i \in I} \mathbf{a}_{i} \phi_{i}+\sum_{i \in I_{H}} \mathbf{b}_{i} H \phi_{i}+\sum_{i \in I_{s}} \mathbf{c}_{i} \chi(r) \psi_{i}(r, \theta)\right\} .
$$




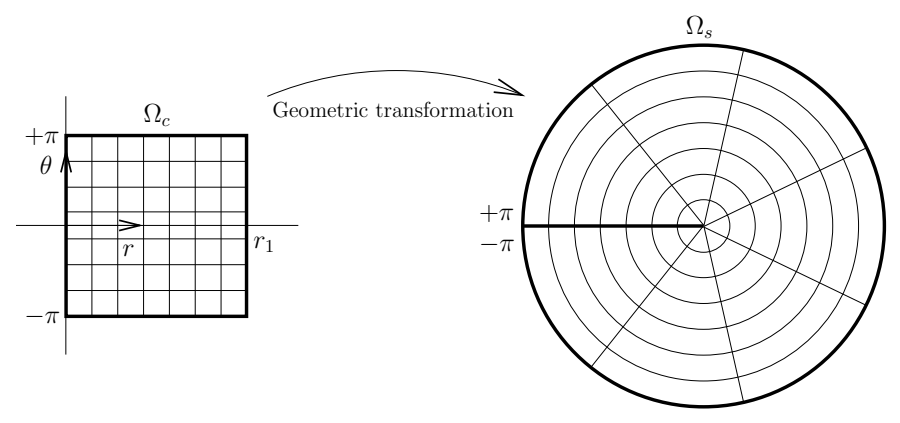

Figure 2. Geometric transformation

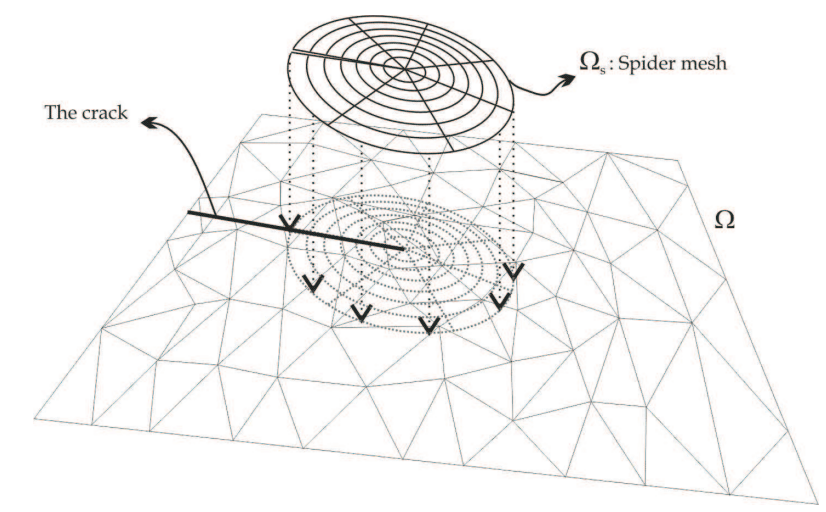

Figure 3. The resulting mesh

The discrete problem reads as

$$
\text { Find } \mathbf{u}^{h} \in \mathbf{V}^{h} \text { such that } a\left(\mathbf{u}^{h}, \mathbf{v}^{h}\right)=l\left(\mathbf{v}^{h}\right) \forall \mathbf{v}^{h} \in \mathbf{V}^{h} .
$$

\section{A priori error estimates}

In order to establish an a priori error estimate, we first define an adapted interpolation operator. It is based, as in (Chahine et al., 2006; Chahine et al., to appear) on a decomposition of the solution $\mathbf{u}$ to Problem [3] as follows:

$$
\mathbf{u}=\mathbf{u}_{r d}+\chi \mathbf{u}_{s} .
$$


The part $\mathbf{u}_{r d}=\mathbf{u}_{r}+(1-\chi) \mathbf{u}_{s}$ is still regular in the sense $\mathbf{u}_{r d} \in H^{2+\varepsilon}\left(\Omega ; \mathbb{R}^{2}\right)$ because $(1-\chi) \mathbf{u}_{s}$ vanishes in a vicinity of the crack tip and is regular elsewhere. The expression of the interpolation operator over the whole domain $\Omega$ is the following:

$$
\Pi^{h} \mathbf{u}=\sum_{i \in I} \mathbf{a}_{i} \varphi_{i}+\sum_{i \in I_{H}} \mathbf{b}_{i} H \varphi_{i}+\sum_{i \in I_{s}} \mathbf{c}_{i} \psi_{i}(r, \theta) \chi(r) .
$$

The coefficients $\mathbf{a}_{i} \in \mathbb{R}^{2}, \mathbf{b}_{i} \in \mathbb{R}^{2}$ are determined by:

if $i \in\left\{I \backslash I_{H}\right\}$ then $\mathbf{a}_{i}=\mathbf{u}_{r d}\left(x_{i}\right)$,

if $i \in I_{H}$ and $x_{i} \in \Omega^{k}(k \in\{1,2\}, l \neq k)$ then $\left\{\begin{array}{l}\mathbf{a}_{i}=\frac{\mathbf{u}_{r d}^{k}\left(x_{i}\right)+\widetilde{\mathbf{u}}_{r d}^{l}\left(x_{i}\right)}{2}, \\ \mathbf{b}_{i}=\frac{\mathbf{u}_{r d}^{k}\left(x_{i}\right)-\widetilde{\mathbf{u}}_{r d}^{l}\left(x_{i}\right)}{2} H\left(x_{i}\right),\end{array}\right.$

where $x_{i}$ denotes the node associated to $\varphi_{i}$, and where it is assumed that there exists a continuation of the crack which splits $\Omega$ into two parts denoted $\Omega^{1}$ and $\Omega^{2}$. The notations $\mathbf{u}_{r d}^{1}, \mathbf{u}_{r d}^{2}$ stand for the restriction of $\mathbf{u}_{r d}$ to $\Omega^{1}$ and $\Omega^{2}$ respectively and $\widetilde{\mathbf{u}}_{r d}^{1}, \widetilde{\mathbf{u}}_{r d}^{2} \in H^{2+\varepsilon}\left(\bar{\Omega} ; \mathbb{R}^{2}\right)$ are some given regular extensions on $\bar{\Omega}$ of $\mathbf{u}_{r d}^{1}$ and $\mathbf{u}_{r d}^{2}$ respectively. The coefficients $\mathbf{c}_{i} \in \mathbb{R}^{2}$ are simply defined by

$$
\mathbf{c}_{i}=r_{i}^{-\alpha} \mathbf{u}_{s}\left(r_{i}, \theta_{i}\right),
$$

where $\left(r_{i}, \theta_{i}\right)$ is the finite element node corresponding to $\psi_{i}$.

An estimate of the regular part is presented in (Chahine et al., 2006; Chahine et al., to appear). It holds for a constant $C>0$ independent of $h$ (for a straight crack):

$$
\left\|\mathbf{u}_{r d}-\Pi^{h} \mathbf{u}_{r d}\right\|_{1, \Omega} \leq C h\left\|\mathbf{u}_{r d}\right\|_{2+\epsilon, \Omega},
$$

where $\|\cdot\|_{s, \Omega}$ stands for the norm of the space $H^{s}\left(\Omega ; \mathbb{R}^{2}\right)$. Thus, it remains to estimate

$$
\left\|\chi \mathbf{u}_{s}-\Pi^{h} \chi \mathbf{u}_{s}\right\|_{1, \Omega}=\left\|\chi\left(\mathbf{u}_{s}-\sum_{i \in I_{s}} \mathbf{c}_{i} \psi_{i}\right)\right\|_{1, \Omega},
$$

which has only a contribution inside the enriched area $\left(r<r_{1}\right)$. The following lemma gives a first estimate of this term.

Lemma 3.1 If $\alpha \geq 1 / 2$, then there exists $C>0$ a constant independent of $h_{c}$ (but which may depend on $r_{0}$ and $\left|\left\|\chi\left|\|=1+\sup _{0 \leq r \leq r_{0}}\right| \chi^{\prime}(r) \mid\right)\right.$ such that denoting $e_{s}=$ $r^{-\alpha}\left(\mathbf{u}_{s}-\sum_{i \in I_{s}} \mathbf{c}_{i} \psi_{i}\right)$ the following estimate holds:

$$
\left\|\chi r^{\alpha} e_{s}\right\|_{1, \Omega} \leq C\left(\left\|e_{s}\right\|_{0, \Omega_{c}}+\left\|r \partial_{r} e_{s}\right\|_{0, \Omega_{c}}+\left\|\partial_{\theta} e_{s}\right\|_{0, \Omega_{c}}\right) .
$$

If $1 / 4 \leq \alpha<1 / 2$, then there exists $C>0$ a constant independent of $h_{c}$ such that the following estimate holds:

$$
\left\|\chi r^{\alpha} e_{s}\right\|_{1, \Omega} \leq C\left(\left\|e_{s}\right\|_{L^{4}\left(\Omega_{c}\right)}+\left\|r \partial_{r} e_{s}\right\|_{L^{4}\left(\Omega_{c}\right)}+\left\|\partial_{\theta} e_{s}\right\|_{L^{4}\left(\Omega_{c}\right)}\right),
$$

where $\|\cdot\|_{L^{4}\left(\Omega_{c}\right)}$ denotes the norm of the space $L^{4}\left(\Omega_{c}, \mathbb{R}^{2}\right)$. 
Proof. One has, for some constant $C>0$ independent of $h_{c}$

$$
\left\|\chi r^{\alpha} e_{s}\right\|_{1, \Omega} \leq C \mid\|\chi\|\|\| r^{\alpha} e_{s} \|_{1, \Omega_{s}} .
$$

But

$$
\begin{aligned}
\left\|r^{\alpha} e_{s}\right\|_{1, \Omega_{s}}^{2} & =\int_{\Omega_{s}}\left(r^{\alpha} e_{s}\right)^{2} d \Omega_{s}+\int_{\Omega_{s}}\left|\nabla\left(r^{\alpha} e_{s}\right)\right|^{2} d \Omega_{s} \\
& =\int_{\Omega_{c}} r^{2 \alpha-1}\left(r\left|e_{s}\right|^{2}+\left|\alpha e_{s}+r \partial_{r} e_{s}\right|^{2}+\left|\partial_{\theta} e_{s}\right|^{2}\right) d r d \theta
\end{aligned}
$$

If $\alpha \geq 1 / 2$ the term $r^{2 \alpha-1}$ can be bounded by $r_{0}^{2 \alpha-1}$ which gives the first estimate and if $1 / 4 \leq \alpha<1 / 2$ the second estimate is obtained thanks to Schwarz's inequality.

\subsection{The regular case}

Standard results on Lagrange interpolation operator (see (Ciarlet, 1978; Ern et al., 2002)) together with Lemma 3.1 lead to the following result.

Proposition 3.1 If $\alpha \geq 1 / 2, f_{i} \in H^{2}\left(0, r_{0}\right), i=1,2$ and $g_{i} \in H^{2}(0,2 \pi), i=1,2$ then the following estimate holds for $e_{s}=r^{-\alpha}\left(\mathbf{u}_{s}-\sum_{i \in I_{s}} \mathbf{c}_{i} \psi_{i}\right)$ :

$$
\left\|\chi r^{\alpha} e_{s}\right\|_{1, \Omega} \leq C h_{c}\|f(r) g(\theta)\|_{2, \Omega_{c}} .
$$

If $1 / 4 \leq \alpha<1 / 2, f_{i} \in H^{2}\left(0, r_{0}\right), i=1,2$ and $g_{i} \in H^{2}(0,2 \pi), i=1,2$ then the following estimate holds:

$$
\left\|\chi r^{\alpha} e_{s}\right\|_{1, \Omega} \leq C h_{c}\|f(r) g(\theta)\|_{W^{2,4}\left(\Omega_{c}\right)},
$$

where $W^{2,4}\left(\Omega_{c}\right)$ is the standard Sobolev space (see (Adams, 1975)).

This result indicates that if the functions $f_{i}, i=1,2$ and $g_{i}, i=1,2$ are some regular functions with respect to $r$ and $\theta$ respectively then the SpiderXfem allows to have an optimal convergence rate provided that $h_{c}$ is of the same order of $h$ (i.e. $\left.\exists \eta>0, h_{c} \leq \eta h\right)$.

Note that in the homogeneous case (constant lamé coefficients), functions $f_{i}, i=$ 1,2 are not necessary. Thus, the functions $p_{j}(r)$ can be omitted in the definition of $\psi_{i}$ [10]. Which means that only a discretization with respect to the variable $\theta$ is necessary. This, of course, greatly reduces the number of degrees of freedom necessary to represent the asymptotic behavior of the displacement. 


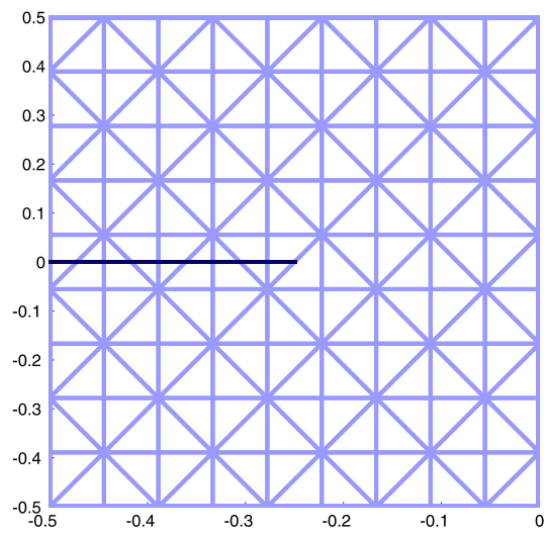

Figure 4. A structured triangulation of the domain $\bar{\Omega}$

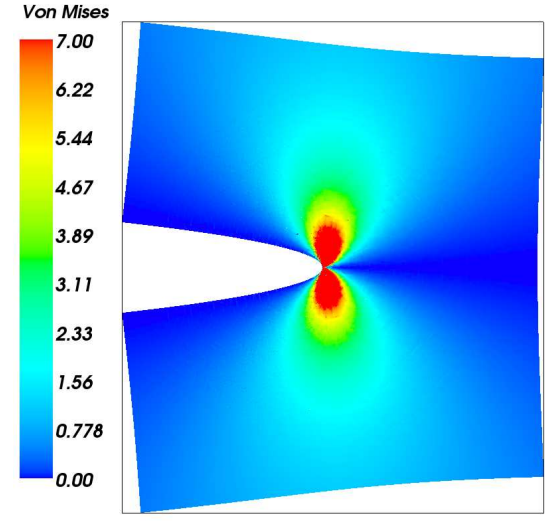

Figure 5. Von Mises stress for the opening mode for an homogeneous material

\subsection{The bi-material case}

In the bi-material case (lamé coefficients having different values from one side of the crack to the other), the typical form of the asymptotic displacement at the crack type is (see (Rice, 1988; Chang et al., 2007) for instance)

$$
\sqrt{r} \sin (\beta \log r) \widetilde{\mathbf{g}}(\theta)+\sqrt{r} \cos (\beta \log r) \overline{\mathbf{g}}(\theta),
$$

where $\widetilde{\mathbf{g}}$ and $\overline{\mathbf{g}}$ are some regular (trigonometric) functions of $\theta$. Unfortunately, the functions $\sin (\beta \log r)$ and $\cos (\beta \log r)$ are not sufficiently regular for the result of the previous section to apply. A rapid analysis allows to note that the term $\left\|r \partial_{r} e_{s}\right\|_{0, \Omega_{c}}$ in Lemma 3.1 only gives a convergence rate of order $\sqrt{h_{c}}$.

The conclusion of this mathematical analysis is that it seems necessary to enrich the SpiderXfem with the whole nonsmooth behavior in $r$. In the bi-material case, both the enrichment with $\sqrt{r} \sin (\beta \log r)$ and with $\sqrt{r} \cos (\beta \log r)$ are necessary. However, once these enrichments are considered, no supplementary dependence of the SpiderXfem in $r$ is necessary, similarly to the previous section. Thus, the necessary enrichment in order to obtain an optimal convergence rate is given by the following definition of functions $\psi_{i}^{k}$ :

$$
\psi_{i}^{1}=\sqrt{r} \sin (\beta \log r) q_{i}(\theta), \quad \psi_{i}^{2}=\sqrt{r} \cos (\beta \log r) q_{i}(\theta) .
$$


The resulting enriched finite element space is

$$
\mathbf{V}^{h}=\left\{\mathbf{u}^{h}=\sum_{i \in I} \mathbf{a}_{i} \phi_{i}+\sum_{i \in I_{H}} \mathbf{b}_{i} H \phi_{i}+\sum_{i \in I_{s}, k=1,2} \mathbf{c}_{i}^{k} \chi(r) \psi_{i}^{k}(r, \theta)\right\}
$$
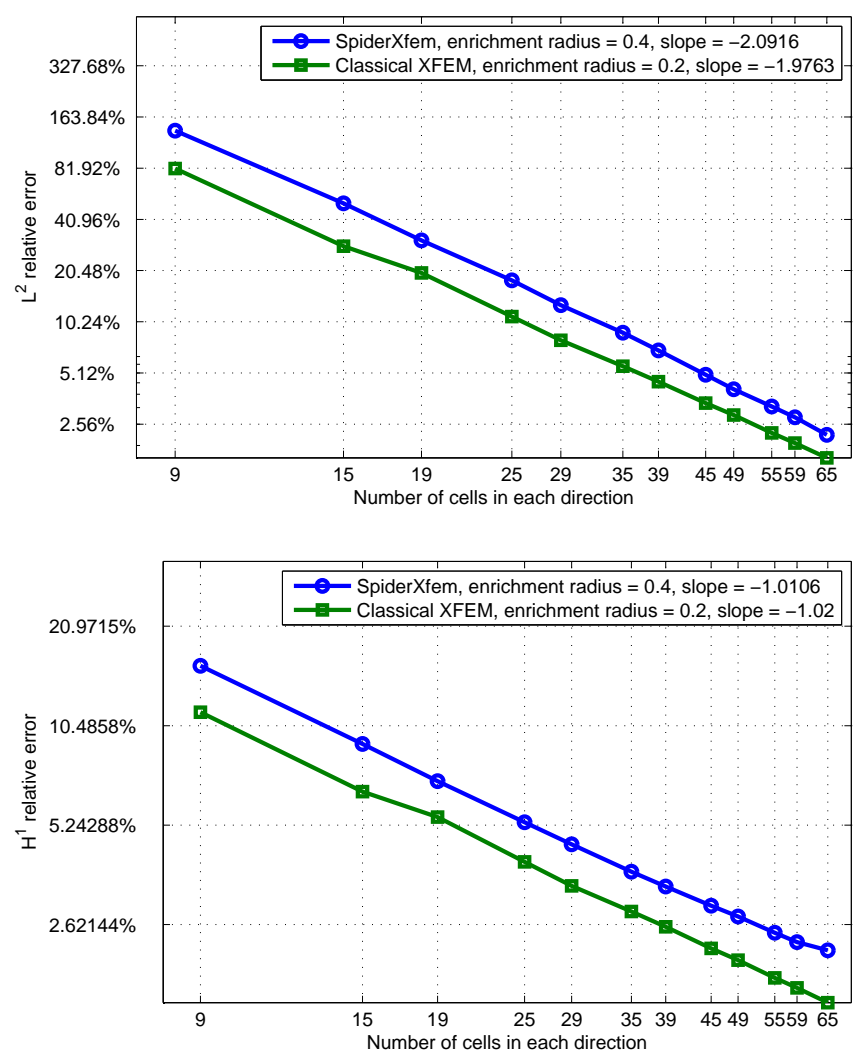

Figure 6. Convergence curves for an isotropic homogeneous cracked material in $L^{2}(\Omega)$-norm and $H^{1}(\Omega)$-norm comparing the SpiderXfem and the classical Xfem. For the SpiderXfem we consider $h_{c}=h / 2$

\section{Numerical experiments}

The computational tests are performed on the simple non-cracked domain $\bar{\Omega}=$ $[-0.5 ; 0.5] \times[-0.5 ; 0.5]$ and with a crack being the line segment $\Gamma_{C}=[-0.5 ; 0] \times\{0\}$. The tests are done for an homogeneous material. The opening mode displacement 
field is the exact solution prescribed as a Dirichlet condition on the domain boundary. This solution is shown on Figure 5. The cut-off function is a piecewise fifth degree polynomial with $r_{0}=0.01$ and $r_{1}=0.4$ (see [8]). The computations are made with the SpiderXfem as described in Section 2 and with the classical Xfem with a fixed enrichment area both over a structured triangulation as depicted on Figure 4. We make the use of Getfem++, our object oriented C++ finite element library (see (Renard et al., http://home.gna.org/getfem/)).

Figure 6 shows the convergence curves for an isotropic homogeneous cracked domain in $L^{2}(\Omega)$-norm and $H^{1}(\Omega)$-norm (energy norm). The two mesh parameters for the SpiderXfem are taken proportional $\left(h_{c}=h / 2\right)$. The convergence rate is optimal for both the two methods. The classical Xfem still gives slightly better results than the SpiderXfem. However, only a partial knowledge of the asymptotic displacement is used with the SpiderXfem. Moreover, the condition number of the stiffness matrix is greatly better in the case of the SpiderXfem as shown on Figure 7.

Figure 8 shows the convergence curve for a bimaterial interface crack in $H^{1}(\Omega)$-norm. The SpiderXfem enrichment considered here is the one given in Section 3.2 and the comparison is done with respect to a refined classical finite element solution. The optimal convergence rate is obtained as in the isotropic homogeneous case even though all the dependency of the singular part of the solution in $\theta$ is approximated.

\section{Concluding remarks}

The presented theoretical and numerical studies emphasize that with the proposed strategy, the dependence in $r$ of the asymptotic displacement has to be known and added to the expression of the enrichment functions. Conversely, the dependence in $\theta$ can be approximated with a one-dimensional finite element. Note that a spectral approximation should also be possible, even if it leads to a more dense stiffness matrix. The coefficients $\alpha$ from [5] and $\beta$ from [24] are determined by a transcendental equation whose solution is $\alpha+i \beta$ (see (Chang et al., 2007) for instance). The advantage of the proposed method is that it is sufficient to find $\alpha$ and $\beta$. Indeed, the search of the complete expression of the asymptotic displacement and the extraction of a base in the classical Xfem approach can be a complex process which is avoided with the SpiderXfem.

The conclusions of sections 3.1 and 3.2 indicate that, in most of the cases, one can make the economy of the finite element method describing the dependence in $r$ of the SpiderXfem (i.e. $p_{j}(r)$ can be omitted in [10]). However, it can also be advantageous to keep it. Indeed, the higher degree nonsmooth modes describing the asymptotic displacement at the crack tip differ from an integer power of $r$ compared to the first one. So, according to the theoretical results, by keeping the finite element method in $r$ the whole asymptotic displacement is optimally approximated, not only the first nonsmooth mode. This could be an interesting property, especially to build higher order finite element methods. 
A perspective to improve the method is to replace the use of a cut-off function by a pointwise or an integral matching condition. This usually leads to a better approximation (see (Laborde et al., 2005)).

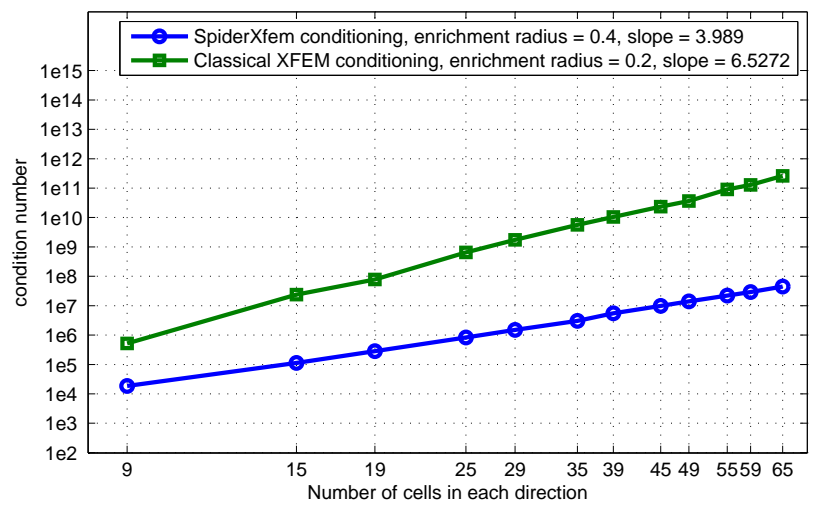

Figure 7. Condition number of the stiffness matrix

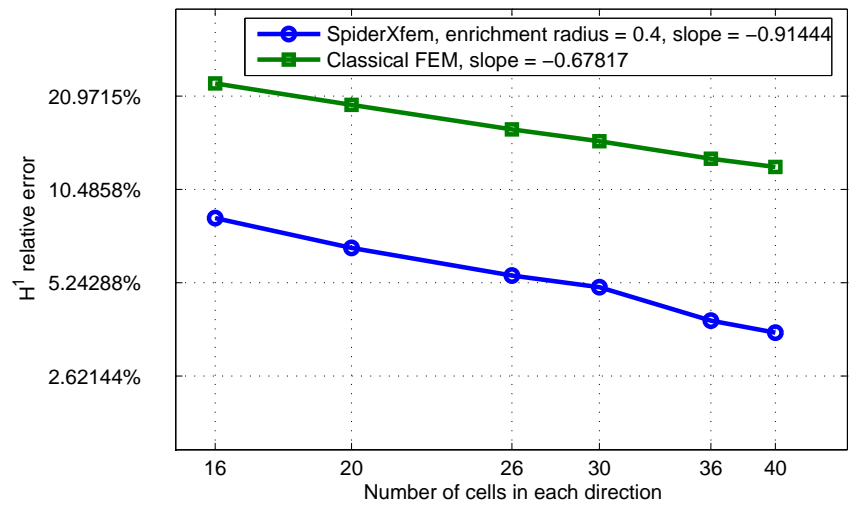

Figure 8. Convergence curves for a bimaterial interface crack in $H^{1}(\Omega)$-norm comparing the SpiderXfem and a classical fem. For the SpiderXfem we consider $h_{c}=h / 2$

Acknowledgements

This work is supported by "l'Agence Nationale de la Recherche", project ANR05-JCJC-0182-01. 


\section{References}

Adams R., Sobolev Spaces, Academic Press, 1975.

Béchet E., Minnebo H., Moës N., Burgardt B., " Improved implementation and robustness study of the X-FEM for stress analysis around cracks", Int. J. Numer. Meth. Engng., vol. 64, p. 1033-1056, 2005.

Bendhia H., "Multiscale mechanical problems : the Arlequin method", C. R. Acad. Sci., série I, Paris, vol. 326, p. 899-904, 1998.

Chahine E., Laborde P., Renard Y., " Quasi-optimal convergence result in fracture mechanics with XFEM", C.R. Math. Acad. Sci. Paris, vol. 342, p. 527-532, 2006.

Chahine E., Laborde P., Renard Y., " Crack tip enrichment in the XFEM method using a cut-off function”, Int. J. Numer. Meth. Engng., to appear.

Chang J., Xu J.-Q., “ The singular stress field and stress intensity factors of a crack terminating at a bimaterial interface", Int. J. Mech. Sci., vol. 49, p. 888-897, 2007.

Ciarlet P., The finite element method for elliptic problems, Studies in Mathematics and its Applications No 4, North Holland, 1978.

Ern A., Guermond J.-L., Éléments finis: théorie, applications, mise en æuvre, Mathématiques et Applications 36, SMAI, Springer-Verlag, 2002.

Glowinski R., He J., Rappaz J., Wagner J., “ Approximation of multi-scale elliptic problems using patches of elements”, C. R. Math. Acad. Sci., Paris, vol. 337, p. 679-684, 2003.

Grisvard P., "Problèmes aux limites dans les polygones - Mode d'emploi”, EDF Bull. Dirctions Etudes Rech. Sér. C. Math. Inform. 1, vol. MR 87g:35073, p. 21-59, 1986.

Grisvard P., Singularities in boundary value problems, Masson, 1992.

Laborde P., Renard Y., Pommier J., Salaün M., “ High Order Extended Finite Element Method For Cracked Domains”, Int. J. Numer. Meth. Engng., vol. 64, p. 354-381, 2005.

Lemaitre J., Chaboche J.-L., Mechanics of Solid Materials, Cambridge University Press, 1994.

Melenk J., Babuška I., “ The partition of unity finite element method: Basic theory and applications”, Comput. Meths. Appl. Mech. Engrg., vol. 139, p. 289-314, 1996.

Moës N., Belytschko T., “ X-FEM: Nouvelles Frontières Pour les Eléments Finis”, Revue européenne des éléments finis, vol. 11, p. 131-150, 1999a.

Moës N., Dolbow J., Belytschko T., “ A finite element method for crack growth without remeshing”, Int. J. Numer. Meth. Engng., vol. 46, p. 131-150, 1999b.

Renard Y., Pommier J., Getfem ++, An open source generic C++ library for finite element methods, http://home.gna.org/getfem/.

Rice J., "Elastic fracture mechanics concepts for interfacial cracks", Journal of Applied Mechanics, vol. 55, p. 98-103, 1988.

Strang G., Fix G., An Analysis of the Finite Element Method, Prentice-Hall, Englewood Cliffs, 1973.

Strouboulis T., Babuska I., Copps K., “ The design and analysis of the Generalized Finite Element Method”, Comput. Meths. Appl. Mech. Engrg., vol. 181, p. 43-69, 2000. 